\title{
Acute hypothalamic suppression significantly affects trabecular bone but not cortical bone following recovery and ovariectomy surgery in a rat model
}

Vanessa R Yingling, Kathryn A Mitchell, Megan Lunny

Background: Osteoporosis is "a pediatric disease with geriatric consequences". Bone morphology and tissue quality co-adapt during ontogeny for sufficient bone stiffness. Altered bone morphology from hypothalamic amenorrhea, a risk factor for low bone mass in women, may affect bone strength later in life. Our purpose was to determine if altered morphology following hypothalamic suppression during development affects cortical bone strength and trabecular bone volume (BV/TV) at maturity. Methods: Female rats ( 25 days old) were assigned to a control (C) group ( $n=45)$ that received saline injections (.2cc) or an experimental group $(\mathrm{GnRH}-\mathrm{a})(\mathrm{n}=45)$ that received gonadotropin releasing hormone antagonist injections (.24mg per dose) for 25 days. Fifteen animals from each group were sacrificed immediately after the injection protocol at Day 50 (C, GnRH-a). The remaining animals recovered for 135 days and a subset of each group was sacrificed at Day 185 [(C$R)(n=15)$ and $(G-R)(n=15)]$. The remaining animals had an ovariectomy surgery $(O V X)$ at 185 days of age and were sacrificed 40 days later (C-OVX) $(n=15)$ and (G-OVX) $(n=15)$. After sacrifice femurs were mechanically tested and scanned using micro CT. Serum Cterminal telopeptides (CTX) and insulin-like growth factor 1 (IGF-1) were measured. Twoway ANOVA [ 2 groups (GnRH-a and Control) X 3 time points (Injection Protocol, Recovery, post-OVX) was computed. Results: GnRH-a injections suppressed uterine weights (72\%) and increased CTX levels by $59 \%$. Bone stiffness was greater in the $\mathrm{GnRH}$-a groups compared to $\mathrm{C}$. Ash content and cortical bone area were similar between groups at all time points. Polar moment of inertia, a measure of bone architecture, was $15 \%$ larger in the GnRH-a group and remained larger than C (19\%) following recovery. Both the polar moment of inertia and cortical area increased linearly with the increases in body weight. Following the injection protocol, trabecular BV/TV was 31\% lower in the $\mathrm{GnRH}$-a group compared to $\mathrm{C}$, a similar deficit in BV/TV was also measured following recovery and postOVX. The trabecular number and thickness were lower in the $\mathrm{GnRH}-\mathrm{a}$ group compared to control. Conclusion: These data suggest that following a transient delay in pubertal onset, trabecular bone volume was significantly lower and no restoration of bone volume occurred following recovery or post-OVX surgery. However, cortical bone strength was 
maintained through architectural adaptations in the cortical bone envelope. An increase in the polar moment of inertia offset increased bone resorption. The current data are the first to suppress trabecular bone during growth, and then add an OVX protocol at maturity. Trabecular bone and cortical bone differed in their response to hypothalamic suppression during development; trabecular bone was more sensitive to the negative effects of hypothalamic suppression. 
1 Acute hypothalamic suppression significantly suppresses trabecular bone but spares

2 cortical bone following recovery and ovariectomy surgery in a rat model.

3

4 Vanessa R. Yingling ${ }^{1,2,3}$, Kathryn Mitchell ${ }^{1}$, Megan Lunny ${ }^{1}$,

$5 \quad{ }^{1}$ Department of Kinesiology, College of Health Professions and Social Work, Temple

6 University, Philadelphia, PA, USA

7 2Department of Anatomy and Cell Biology, Temple University, School of Medicine,

8 Philadelphia, PA, USA

9 '3epartment of Kinesiology, California State University East Bay, Hayward, CA, USA

11 Vanessa R. Yingling

12 Department of Kinesiology

13 California State University East Bay

1425800 Carlos Bee Boulevard

15 Hayward, CA 94542

16 Vanessa.yingling@csueastbay.edu 


\section{Introduction}

Osteoporosis is "a pediatric disease with geriatric consequences" (Golden, 2000). Bone morphology and tissue quality co-adapt during ontogeny for sufficient bone strength (Jepsen, 2011). Suboptimal bone strength in individuals who do not reach peak bone mass during childhood or adolescence may contribute to the development of fractures later in life (Bachrach, 2001). Therefore, factors during the developmental years such as, hypothalamic amenorrhea, a risk factor for low bone mass in women, may affect bone strength later in life.

The timing of puberty has emerged as a crucial factor in bone strength development. Peak bone mineral accrual rate occurs at puberty (Warren \& Stiehl, 1999), with an accrual of $26 \%$ of adult total bone mineral within 2 years of pubertal onset (Pitukcheewanont et al., 2013). However, a delay in the timing of puberty is one factor among many that correlate with low bone mass in young women (Nattiv et al., 1994; Wiggins \& Wiggins, 1997; Yingling \& Khaneja, 2006). Warren et al. (2002) found the positive correlation between the age of menarche and stress fracture occurrence to be stronger than the age of menarche and bone mineral density (BMD) (Warren et al., 2002). Yet the effect of a delayed puberty on peak bone strength at maturity particularly post-menopause when estrogen levels in women decrease and bone mass is lost remains unclear. Young women with delayed pubertal onset may not build a strong skeleton and be at risk for fracture in the short term and later in life, in particular postmenopause. 
Multiple factors affect the structural development of the skeleton; in particular estrogen levels during growth are an important factor in the pathogenesis of bone fragility (Drinkwater et al., 1984). The delay of menarche and infrequent menstrual cycles decrease estrogen levels during adolescence and decrease peak bone mass (Myerson et al., 1992; Warren et al., 2003; Nattiv et al., 2007). Investigators have identified bone densities in young (17-35 yr) athletic women with decreased estrogen levels similar to the bone densities of 51-year-old women (Myerson et al., 1992; De in females by endosteal apposition. Low estrogen levels during growth may result in a thinner cortex if the increased endosteal resorption (De Crée, 1998) is not offset by an increased periosteal apposition (Loucks, Verdun \& Heath, 1998). The relative cellular activity on these bone surfaces affects bone size, a critical element of bone strength. In trabecular bone, post-menopausal women have significantly lower bone volume and less trabeculae (Akhter et al., 2007). Trabecular number and thickness are decreased following ovariectomy surgery, a model of post-menopausal bone loss, in both mature and young animals (Wronski, Dann \& Horner, 1989; Wronski et al., 1989; Sims et al., 1996; Roux et al., 1996; Lane et al., 1998; Bourrin et al., 2002). Animal models of low estrogen (hypothalamic suppression) during adolescence report deficits in cortical bone strength (Yingling \& Khaneja, 2006; Yingling \& Taylor, 2008) and trabecular structure (Rakover et al., 2000; Yingling et al., 2007) and therefore it is important to determine if these deficits remain and whether they are exacerbated during the menopause. Specifically, the long term effects of hypothalamic suppression during development at a 
66 time point at maturity, in particular following estrogen loss due to the menopause

67 modeled by ovariectomy surgery needs to be investigated.

68

The purpose of this study was to investigate the effect of a delay in puberty on bone strength and structure immediately post-puberty and at maturity in female rats. This model offers an opportunity to reproduce an environment of delayed puberty and to investigate the effect on bone strength and structure at critical time points throughout the life span. The investigative hypothesis predicts that administration of a $\mathrm{GnRH}$ antagonist prior to the onset of the first estrus cycle would suppress the increase in estrogen levels associated with the onset of puberty and impede the development of cortical and or trabecular bone strength and structure acutely and at maturity.

\section{Methods}

\section{Animal Protocol}

Female rats ( 25 days) (Charles Rivers Laboratories, Wilmington, MA, USA) were assigned to a control $(C)$ group $(n=45)$ that received saline injections $(.2 c c)$ for 25 days or an experimental group $(\mathrm{GnRH}-\mathrm{a})(\mathrm{n}=45)$ that received gonadotropin releasing hormone antagonist injections (.24mg per dose) (My Biosource, San Diego, CA, Luteinizing Hormone Releasing Hormone [Nal-Glu] Antagonist, Peptide (\#MBS659559)) (Figure 1). The dosage ranged from $5.0 \mathrm{mg} / \mathrm{kg}$ to $1.25 \mathrm{mg} / \mathrm{kg}$ throughout the protocol as the animal body weights increased. Fifteen animals from each group were sacrificed immediately after the injection protocol at Day $50(\mathrm{C}, \mathrm{GnRH}-\mathrm{a})$. The remaining animals recovered from the saline or $\mathrm{GnRH}-\mathrm{a}$ injections for 135 days and a subset of each group was sacrificed at Day $185[(C-R)(n=15)$ and $(G-R)(n=15)]$. The remaining animals 
89

90

91

92

93

94

95

96

97

3

had an ovariectomy surgery (OVX) at 185 days of age and were sacrificed 40 days later (COVX)(n=15) and (G-OVX) (n=15). After sacrifice (50, 185 and 225 days) (n=15/group), femurs and tibia were mechanically tested and femurs were scanned using micro CT and serum Cterminal telopeptides (CTX) and insulin-like growth factor 1 (IGF-1) were measured.

4

All animals were given food and water ad libitum. All animals were monitored daily for vaginal opening, an indicator of pubertal onset (Ojeda et al., 1976). Body weights were taken daily until the onset of puberty and then every 4 days. Growth rates were calculated (grams/week) for early puberty (weight day 37-weight day 23)/2) and late puberty (weight day 50-weight day 37)/2); puberty typically occurs at day 30 . On the day of sacrifice, body weight was measured. Animals were anesthetized using $\mathrm{CO}_{2}$ (Toft, Petersen, Dragsted, \& Hansen, 2006) and blood was taken via a cardiac puncture. The animals were then killed by an overdose of $\mathrm{CO}_{2}$. Uterus, ovaries, retroperitoneal and gonadal fat pads, and triceps surae muscle tissue were collected and weighed. Femora and tibiae were removed and cleaned of soft tissue. The right femora and tibiae were mechanically tested and ashed for bone mineral content while the left femora were fixed in $10 \%$ buffered formalin for 48 hours to one week for micro-CT imaging. All procedures were approved by the Institutional Animal Care and Use Committee (IACUC) at Temple University (3396). Gonadotropin-releasing hormone antagonists (GnRH-a) injections have successfully delayed the onset of puberty by hypothalamic suppression resulting in low estrogen levels in female rats and have the advantage that normal hypothalamicpituitary function is restored after cessation of injections (Roth et al., 2000). 
114 Mechanical Testing

115 Mechanical strength of the right femora and tibiae was measured under three-point

116 bending using a materials testing machine (Bose Electroforce, Eden Prairie, MN)

117 containing a $450 \mathrm{~N}$ load cell. Bones were placed on the lower supports anterior side

118 down and loaded in the anterior-posterior plane. Span length of the lower supports was

119 maximized to minimize the effect of shear loading. Span lengths were as follows for

120 each time point: injection protocol (femur-16.8 $\mathrm{mm}$, tibia-22.3mm), recovery (femur-

$12121.7 \mathrm{~mm}$, tibia-26.3mm), post-OVX (femur-20.2 mm, tibia-25.2mm). Prior to testing the

122 bones were thawed in saline to ensure hydration and then loaded to failure at a rate of

$1230.05 \mathrm{~mm} / \mathrm{sec}$. During testing, force and displacement data were collected. Bending

124 moment was calculated from the force data $(M=F L / 4)(\mathrm{Nmm})$ and displacement data

125 were divided by $\left(L^{2} / 12\right)\left(\mathrm{mm} / \mathrm{mm}^{2}\right)$ where $L$ is the distance between the lower supports

126 (span length). Mechanical properties of the whole bone were then determined from the

127 moment vs. normalized displacement curves. Mechanical properties included: peak

128 moment $(\mathrm{Nmm})$ (the maximal load the specimen sustained) and stiffness ( $\left.\mathrm{Nmm}^{2}\right)($ slope

129 of the initial linear portion of the moment-displacement curve). Both the peak moment

130 and the stiffness are measures of bone structural strength and stiffness and both

131 measurements are influenced by the bone material/composition measured by ash

132 fraction and the distribution of that material (bone geometry) measured by polar moment 133 of inertia.

134

135 Micro-CT Analysis 
The left femora were fixed in $10 \%$ buffered formalin for 48 hours to one week, stored

137 in $70 \%$ ethanol, and scanned in an ex vivo $\mu \mathrm{CT}$ scanner (SkyScan 1172; SkyScan, Aartslaar, Belgium) to measure both geometrical changes in the cortical bone sites and

139 structural changes in trabecular and cortical bone sites. The Skyscan-1172 has a sealed micro focus $\mathrm{X}$-ray tube that emits from $20 \mathrm{keV}$ to $100 \mathrm{keV}$ energy with a 10 megapixel (4000x2096), 12-bit cooled CCD camera. Scanning was performed using a source voltage of $60 \mathrm{kV}$ and source current of $167 \mu \mathrm{A}$ with a $0.5 \mathrm{~mm}$ Al filter to minimize the beam hardening from the polychromatic nature of the sealed X-ray source. Scans were done with a rotation step of $0.4^{\circ}$ through $180^{\circ}$ and a pixel size of $7.7 \mu \mathrm{m}$. The Feldkamp cone-beam reconstruction algorithm was used to reconstruct the three146 dimensional cross sections along with addressing the ring artifact reduction of 10 and beam hardening corrections of $20 \%$. Approximately 400 slices in both the metaphyseal 148 trabecular and mid-diaphyseal cortical regions were analyzed. Region of interest for the trabecular bone in the distal femora were positioned at one image distal to the point that

150 the growth plate disappeared to quantify metaphyseal trabecular bone. Cortical region 151 of interest was taken from the midshaft of the femur (200 slices above and below).

152 Trabecular bone analysis included percent bone volume (BV/TV) (volume of bone 153 relative to the total volume of the region of interest), trabecular thickness (Tb.Th), 154 trabecular number (Tb.N), trabecular separation (Tb.S), while cortical bone analysis 155 included total cross-sectional area (T.Ar), cortical bone area (Ct. Ar), marrow area 156 (Ma.Ar), cortical thickness (Ct.Th), cortical volume fraction (BV/TVcort), polar moment of 157 inertia (J). All parameters were calculated according to the ASBMR standards (Parfitt et 158 al., 1987; Bouxsein et al., 2010). 
Bone Mineral Content

After mechanical testing, the right femora were flushed with phosphate-buffered saline to discard the marrow. Dry weight of the bones was determined after drying in an oven at $100^{\circ} \mathrm{C}$ for $24 \mathrm{hr}$. Ash weight was determined after ashing the bone in a muffle furnace (Fischer Scientific) at $700^{\circ} \mathrm{C}$ for $24 \mathrm{hr}$. Ash fraction was calculated as ash weight / dry weight.

\section{Blood Chemistry}

C-terminal telopeptides or carboxy-terminal collagen crosslinks (CTX) were measured in serum using an immunoenzymometric assay (Rat-Laps EIA, Immunodiagnostic Systems Inc., Fountain Hills, AZ, USA). The detection limit of the assay was $2.0 \mathrm{ng} / \mathrm{mL}$. Serum insulin-like growth factor 1 (IGF-1) was measured using an immunoenzymometric assay (Rat/Mouse IGF-1, Immunodiagnostic Systems Inc., Fountain Hills, AZ, USA). The sensitivity of the assay was $63 \mathrm{ng} / \mathrm{mL}$.

\section{Data Analysis}

Two-way ANOVA [2 groups (GnRH-a and control) $\times 3$ time points (Injection Protocol, Recovery, post-OVX) was computed to determine interactions between the $\mathrm{GnRH}-\mathrm{a}$ and control groups for the dependent variables. A Sidak's multiple comparison test was used to determine significant interactions between groups and time points. A level of $p \leq 0.05$ was considered significant. The relationship of body weight to polar moment of inertia and cortical area were determined by regression analysis $(p<0.05)$. 
182 The relationship of polar moment of inertia to cortical area was determined by

183 regression analysis $(\mathrm{p}<0.05)$. All statistical analyses were performed in GraphPad

184 (GraphPad Prism version 6.00 for Windows, GraphPad Software, San Diego California

185 USA). Mechanical variables were normalized with a linear regression-based correction

186 using body weight (Di Masso et al., 1997) since at sacrifice there was a significant

187 difference in body weight between the groups (non-normalized values are also

188 presented). All variables with an $\mathrm{R}^{2}$ level greater than 0 were normalized to avoid

189 choosing an arbitrary $\mathrm{R}^{2}$ value as a cut-off for normalization.

\section{Results}

Vaginal Opening- Delayed Puberty

A significant delay in vaginal opening (an indicator of the onset of puberty) was evident following the 25-day $\mathrm{GnRH}$-a injection protocol in all $3 \mathrm{GnRH}$-a groups ( $\mathrm{GnRH}$ -

a, G-R, G-OVX) (Figure 2). Only 40-60\% of the GnRH-a groups had a vaginal opening by day 50 , the end of the injection protocol (Figure 2). The average day for VO in the control groups (C, C-R, C-OVX) was 34 days of age. The G-R and G-OVX groups had $100 \%$ of the group reach puberty, indicated by a vaginal opening, by 60 days of age, almost $2 x$ later than the control groups.

Anthropometrics Uterine weights were $72 \%$ lower in the $\mathrm{GnRH}$-a group compared to control a further indication of the success of the $\mathrm{GnRH}-$ a injection protocol (Figure 3A). Uterine 
204 weight recovered during the recovery phase, there was no difference between the C-R

205 and G-R groups (Figure 3A). Uterine weights were approximately $60 \%$ lower following

206 the OVX surgery in both groups; C-OVX and G-OVX (Figure 3A). The hypothalamic

207 suppression via $\mathrm{GnRH}-a$ injections significantly increased the growth rates of the

$208 \mathrm{GnRH}$-a groups from week 5 to 7 (late puberty) compared to control (Figure 3B).

209 Growth rates in the control groups significantly slowed down weeks 5 to 7 (late puberty)

210 compared to weeks 3 to 5 (early puberty) (Figure 3B). There was a significant group

211 effect for body weight at sacrifice $(p=0.0015)$. The $\mathrm{GnRH}$-a groups were significantly

212 heavier than controls. Body weights increased at each time point, recovery and post-

213 OVX (Figure 3C).

214 Blood Chemistry

215 There was a significant interaction between group and time point for CTX, a

216 marker of bone resorption, indicating that differences between groups occurred at

217 different time points. CTX levels for the GnRH-a group were significantly higher (59\%)

218 compared to control following the 25-day injection protocol (Figure 4A). Simple main

219 effects of time point within controls was significant, the CTX levels were the lowest at

220 the recovery time point with no differences between acute and post OVX levels.

221 However the CTX levels for the $\mathrm{GnRH}$-a group were the highest during the acute

222 treatment phase even higher than levels post-OVX (Figure 4A). A significant interaction

223 was also found for IGF-1 serum levels. No differences were found between GnRH-a

224 and C following the injection protocol or recovery period, however IGF-1 levels were

$22533.3 \%$ lower in the $\mathrm{GnRH}$-a group between the injection protocol and the recovery stage

226 (Figure 4B). 
Mechanical Properties

229

There was a significant interaction between group and time point for peak

moment of the femur. The G-R group was $28 \%$ stronger following recovery compared to

C-R (Table 1). However, no differences in peak moment between GnRH-a and C

groups were found following the injection protocol or post-OVX (Table 1). Stiffness in

the femur was significantly greater in the $\mathrm{GnRH}-\mathrm{a}$ groups compared to control. No group differences in mechanical strength were found in the tibia (Table 1).

Micro-CT Analysis-Cortical

The polar moment of inertia $(\mathrm{J})$ of the femur was significantly larger in the $\mathrm{GnRH}-$ a groups compared to control. In addition, cortical area, the amount of bone, trended higher $(p=0.0619)$ in the $\mathrm{GnRH}-a$ groups. The total area, marrow area and cortical

240 thickness were not significantly different between groups. Polar moment of inertia

241 increased with later time points compared to the first time point following the injection

242 protocol (Table 1). The cortical area did significantly increase with each time point with

243 the greatest cortical area being post OVX in both groups $(p<0.0001)$. Marrow area

244 (Ma.Ar) decreased significantly in both groups from the post injection protocol time point to the recovery but remained constant from recovery to post OVX ( $p<0.0001)$. Total area (T.Ar) was greater post OVX compared to the acute time point but no differences were detected between the acute and recovery time points. Cortical thickness was significantly thinner at the acute time point compared to recovery and post OVX. 
250

251

252

253

254

255

256

257

258

259

260

261

262

263

264

265

266

267

268

269

270

271

Micro-CT Analysis-Trabecular

Both trabecular bone volume and structure were acutely affected by the GnRH-a injection protocol and these deficits lasted through maturity and post-OVX. GnRH-a group had less percent bone volume (BV/TV) at each time point compared to control. Following the injection protocol, BV/TV was 31\% lower in the $\mathrm{GnRH}$-a group compared to $\mathrm{C}$, a similar deficit in BV/TV was also measured following recovery and post-OVX. The simple main effect of time point within each group ( $\mathrm{GnRH}-\mathrm{a}$ and Control) were significant, the greatest BV/TV for both groups was post recovery; the two other time points were less but post-OVX was greater than the BV/TV following the injection protocol (Figure 5A). The greater BV/TV in the control groups was due to both a larger trabecular number and a thicker trabecula. The trabecular number was greater in the control group compared to GnRH-a but both groups had the greatest number of trabecula at recovery and lower numbers post-OVX (Figure 5B). Following the injection protocol the control group had a third greater number of trabeculae, the larger number continued at recovery and post-OVX. The trabeculae were also thicker in the control groups compared to $\mathrm{GnRH}-a$ groups. The trabeculae were thickest at recovery and thinnest following the injection protocol (Figure 5C). On average, the trabeculae of the control groups were 3\% thicker than the $\mathrm{GnRH}$-a groups. The lowest trabecular separation was measured at recovery but after the OVX surgery the trabecular separation increased (Figure 5D).

\section{Bone Mineral Content}


The ash fraction was significantly less following the injection protocol compared to recovery and post-OVX time points in both groups. No difference in ash fraction was

274 found between the $\mathrm{C}$ and GnRH-a groups.

Body Composition

Triceps Surae muscle mass per body weight (\%) was significantly different depending on group (interaction). However, post-hoc tests did not indicate difference between groups at any of the time points (Table 1). The total weight for the retroperitoneal and gonadal fat pads were trending larger in the $\mathrm{GnRH}$-a groups $(p=0.064)$. Total fat pad weights were significantly higher than the injection protocol in both the control and GnRH-a groups at recovery and post-OVX (Table 1).

\section{Efficiency of Bone Structure}

Both groups (Control and $\mathrm{GnRH}-\mathrm{a}$ ) built competent structures during the injection protocol, during recover and post-OVX. The polar moment of inertia increased linearly with the increases in body weight and no differences in slope or intercepts were detected between groups (Figure 6A). In addition, cortical area (Ct.Ar) also increased with body weight in a similar manner for both groups (Figure 6B). The efficiency of the bone structure expressed by the regression of cortical area and polar moment of inertia

291 indicated that as the cortical area increased so did the polar moment of inertia. The 292 larger polar moment of inertia mechanical strength of the femoral diaphysis increased. 
293 The relationship between cortical area and polar moment of inertia were not different

294 between groups (Figure 6C).

\section{Discussion}

Previously we reported a short term deficit in bone strength following hypothalamic suppression during growth (Yingling \& Khaneja, 2006; Yingling \& Taylor, 2008) with cortical bone strength recovering by 6 months of age (Yingling \& Khaneja, 2006). In the current study, bone structure and strength were assessed at maturity following ovariectomy (OVX) surgery while still maintaining the acute hypothalamic suppression via gonadotropin releasing hormone antagonist injections during growth; thus investigating the response of both cortical and trabecular bone growth on bone strength, geometry and mineral at maturity, particularly after the menopause. The hypothesis that environment during bone development affects the structure at maturity was partially supported by our data. Functional bone strength was maintained through architectural adaptations in the cortical bone envelope throughout the lifespan however, a lower trabecular bone mass during growth was retained through recovery and post ovariectomy surgery. Our data support the hypothesis that trabecular bone is more vulnerable to factors negatively affecting growth and are less likely to recover from 311 these deficits.

An acute deficit in bone strength (cortical diaphysis) was not found the in current 314 study following a delayed pubertal onset, hypothalamic suppression. In fact, following 
315 recovery the femoral peak moment was greater in the $\mathrm{GnRH}$-a group (G-R) compared

316 to control (C-R). The cortical bone architecture specifically the polar moment of inertia

317 was greater in the $\mathrm{GnRH}$-a groups which rescued bone strength following the delayed

318 puberty. Cortical bone stiffness increased with aging (recovery and post-OVX).

319 However, trabecular bone volume (BV/TV) was significantly lower in the GnRH-a groups

320 compared to control immediately following the injection protocol, after recovery and post

321 OVX surgery. Both trabecular number and thickness were lower for all time points as

322 well.

323

324

GnRH-a injections successfully suppressed hypothalamic function

325 The GnRH-a injections effectively suppressed hypothalamic function as indicated

326 by the delay in the age of vaginal opening, an indicator of puberty in the rat. In addition,

327 uterine weights in the $\mathrm{GnRH}$-a groups where on average $72 \%$ lower compared to

328 control groups. Suppression of the hypothalamus lowers GnRH secretion which in turn

329

suppresses luteinizing hormone $(\mathrm{LH})$ and follicle stimulating hormone $(\mathrm{FSH})$ and thus

330 suppresses estrogen secretion from the ovaries and lowers serum estrogen levels

331 (Schally, 1970). Lower uterine weights are indicative of suppressed estrogen release

332 from the ovaries. Although estrogen levels were not measured in the current study,

333 previous data corroborates suppressed serum estradiol levels using the $\mathrm{GnRH}-\mathrm{a}$

334 injection protocol in pre-pubescent female rats (Yingling \& Khaneja, 2006; Yingling et

335 al., 2007; Yingling \& Taylor, 2008; Saine et al., 2011). In addition, serum CTX levels

336 were significantly increased by $59 \%$ in the $\mathrm{GnRH}-\mathrm{a}$ group an indicator of increased

337 bone resorption also associated with suppressed estrogen secretion. 
339 Trabecular bone volume significantly lower in the GnRH-a groups

The increase in bone resorption indicated by increased CTX levels accompanied

341 a $31 \%$ lower trabecular bone volume (BV/TV) in the GnRH-a groups compared to

342 control immediately following the injection protocol. The deficit in trabecular bone

343 volume was not improved during the recovery period and following OVX surgery, a $34 \%$

344 deficit in trabecular BV/TV remained. We have previously reported a lower yet not

345 statistically significant trabecular volume via histomorphometry using a similar $\mathrm{GnRH}-\mathrm{a}$

346 dosage (Yingling et al., 2007) Yet using texture analysis, lower BV/TV at specific

347 orientations was found and remained lower at maturity, specifically in orientations that

348 do not resist loading (Yingling et al., 2007). Long-term suppression of trabecular bone

349 was also reported by Rakover et al. (2000) using peripheral quantitative computerized

350 tomography (pQCT) and dual energy X-ray absorptiometry (DXA) (Rakover et al.,

351 2000). Trabecular bone catch-up growth was not evident in the GnRH-a group,

352 however, the changes in trabecular BV/TV over time were similar between groups.

353 These data suggest that following a transient delay in pubertal onset, the growth

354 patterns remain similar in both $\mathrm{GnRH}-\mathrm{a}$ and control animals with no restoration of

355 trabecular bone volume in the distal femur. Similar failures to regain bone mass have

356 been reported in elite female athletes following delayed puberty and amenorrhea during

357 young adulthood (Warren et al., 2002, 2003), specifically amenorrheic dancers receiving

358 hormone replacement therapy for 2 years had no increase in bone mineral density

359 (BMD) compared to placebo or control groups. 
Reduced trabecular bone volume was primarily due to fewer trabeculae following

362 the injection protocol (29\% less); the thickness of the trabeculae was only $3-5 \%$ thinner

363 in the GnRH-a groups. In osteoporotic women, trabecular bone is typically reduced due

364 to trabecular thinning and loss of trabecular connectivity (Aaron, Makins \& Sagreiya,

365 1987; Borer, 2005). A previous study using the $\mathrm{GnRH}-\mathrm{a}$ protocol at a $100 \mu \mathrm{g} / \mathrm{day}$

366 dosage measured a loss in percent trabecular bone volume (BV/TV) due to a larger

367 trabecular separation and lower trabecular number (Yingling et al., 2007). The current

368 data are the first to suppress trabecular bone during growth, and then add an OVX

369 protocol at maturity; the result was a lower trabecular number and a continued thinning

370 of the trabeculae throughout the life span. Further investigation is needed to determine

371 if this pattern would be replicated in the vertebral spine and other trabecular bone

372 regions.

373

Cortical bone strength preserved following GnRH-a Injections

Interestingly while acquisition of trabecular bone volume was reduced, there were no differences in cortical bone area $(p=0.06)$, total area or marrow area, only the polar moment of inertia differed significantly between groups. Cortical bone adapted to

378 the acute $\mathrm{GnRH}-\mathrm{a}$ injection protocol during development by varying the architecture within the diaphysis to maintain a sufficiently strong bone. Cortical bone strength is maintained throughout the aging process by a coordination of morphological and compositional traits including cortical area, geometry and tissue mineralization. These

382 traits can co-vary in order to build a functional bone structure (Jepsen et al., 2013;

383 Schlecht \& Jepsen, 2013). The GnRH-a injections did not affect mineralization as 
384 measured by ash fraction in the current study. The bone mass measured by cortical

385 area was trending higher in the $\mathrm{GnRH}$-a groups yet was correlated with the increased

386 body weight (Figure 6B). Bone geometry measured by polar moment of inertia

387 (distribution of bone mass in the cortical diaphysis) was greater resulting in greater

388 femoral stiffness in the $\mathrm{GnRH}$-a groups due to increase in bone distributed away from

389 the bending axis. The cortical diaphysis was able to maintain optimal structure during

390 the period of hypothalamic suppression and even increase strength during recovery due

391 to the larger polar moment of inertia.

392

393

Body weight is commonly increased following $\mathrm{GnRH}-a$ injections (Rakover et al.,

394 2000; Roth et al., 2000; Yingling \& Taylor, 2008) and is due to increased growth rates

395 during late puberty. Increased growth rates have been associated with strength deficits

396 (Rawlinson et al., 2009) potentially due to increased woven bone growth on the

397 periosteal surface. Although bone formation and woven bone were not measured in this

398 study there were no changes in ash fraction, a measure of mineralized bone. Both

399 cortical area and polar moment of inertia correlated with the larger body weights in the

$400 \mathrm{GnRH}$-a groups indicating that the change in body size was matched with appropriate

401 bone structural changes in the current study. The cortical area was also correlated with

402 the polar moment of inertia an indication of structural efficiency, as bone mass

403 increases so should the distribution of that mass in order to increase bone strength. All

404 correlations between bone variables and body weight were similar between the control

405 and $\mathrm{GnRH}$-a groups.

406 
The dosage of the GnRH-a injections and timing of injections may affect the short

408 term effects on the cortical bone strength. One explanation for the maintenance of

cortical bone strength following the injection protocol may be moderate estrogen

410 suppression in the current study. Approximately $50 \%$ of all animals in the $\mathrm{GnRH}-\mathrm{a}$

411 group reached puberty by day 50 , the end of the injection protocol. Previous studies

412 using a similar dose of $\mathrm{GnRH}$-a reported lower estrogen levels (27\%) following a $\mathrm{GnRH}-$

413 a injection protocol (Rakover et al., 2000; Yingling \& Khaneja, 2006; Yingling \& Taylor,

414 2008) but the subset of animals that never reached pubertal onset had a greater

415 suppression of estrogen (50\%) indicating that complete delay of puberty reduces

416 estrogen to a larger extent and may have greater effects on cortical bone structure

417 (Yingling \& Khaneja, 2006).

418

These data in the current study suggest a difference in response to hypothalamic suppression (GnRH-a injections) between cortical and trabecular bone. These data suggest that even as serum bone resorption levels increased, cortical bone structure was altered to preserve bone strength and function. The difference in cortical and trabecular bone sensitivity to estrogen suppression has also been reported in human studies of women with oophorectomy (Genant et al 1982) who needed a 4-fold higher dose of estrogen to prevent trabecular bone loss compared to cortical bone loss. One theory is that trabecular bone has more ER $\beta$ (Bord et al., 2001; Khosla, 2008). 
430

431

432

433

434

435

436

437

438

439

440

441

442

443

444

445

446

447

448

449

450

451

452

Limitations of the GnRH-a injection model

Multiple variables will affect skeletal development during a delay in puberty

including the age at which the delay occurs, the duration of delay and the severity of the delay. To understand fully the mechanisms that affect mechanical properties due to a delay in pubertal development and the long term consequences of such a delay necessitates an animal model that reflects closely the clinical characteristics of delayed puberty. The $\mathrm{GnRH}$-a model does replicate key factors reported clinically in patients with delayed puberty. Uterine and ovarian weights were significantly suppressed suggesting that estradiol levels are suppressed in animals using this model similar to human athletes with primary and secondary amenorrhea (Drinkwater et al., 1984; Warren et al., 1991; Pettersson et al., 1999). However, there are limitations to this model. The increased body weights are not consistent with clinical populations. The $\mathrm{GnRH}$-antagonist model of delayed pubertal development only affects the hypothalamic-pituitary-gonadal axis and clinically the condition of delayed pubertal onset may be a more complex interaction of somatic and reproductive maturation. Studies that treated amenorrheic dancers for 2 years with hormone replacement therapy found no difference in BMD between treated and placebo groups (Cumming, 1996; Warren et al., 2003) suggesting that estradiol is not the only factor in the dancer's bone loss.

\section{Conclusion}

Following a delay in pubertal onset during development, functional bone strength was maintained through architectural adaptations in the cortical bone envelope 
453 throughout the lifespan however, a lower trabecular bone mass during growth was

454 retained through recovery and post ovariectomy surgery. An increase in the polar

455 moment of inertia offset increased bone resorption. Cortical bone adapted to an acute

456 environmental insult by varying the architecture within the diaphysis to maintain a

457 sufficiently strong bone. The acquisition of trabecular bone volume was reduced after

458 the injection protocol that delayed pubertal onset trabecular bone volume was not

459 restored following recovery and remained lower post OVX surgery. The data suggest

460 that, trabecular bone was more sensitive to changes in the systemic effects resulting

461 from hypothalamic suppression and bone volume was not restored following recovery.

462

463 Acknowledgements: We thank the members of the Skeletal Adaptation and

464 Development laboratory in the Department of Kinesiology at Temple University.

\section{References}

Aaron, J. E., Makins, N. B., \& Sagreiya, K. (1987). The microanatomy of trabecular bone loss in normal aging men and women. Clinical Orthopaedics and Related Research, (215), 260-271.

Akhter, M. P., Lappe, J. M., Davies, K. M., \& Recker, R. R. (2007). Transmenopausal changes in the trabecular bone structure. Bone, 41(1), 111-116. http://doi.org/10.1016/j.bone.2007.03.019

Bachrach, L. K. (2001). Acquisition of optimal bone mass in childhood and adolescence. Trends in Endocrinology and Metabolism: TEM, 12(1), 22-28. 
475 Bord, S., Horner, A., Beavan, S., \& Compston, J. (2001). Estrogen receptors alpha and 476 beta are differentially expressed in developing human bone. The Journal of Clinical Endocrinology and Metabolism, 86(5), 2309-2314. http://doi.org/10.1210/jcem.86.5.7513

Borer, K. T. (2005). Physical activity in the prevention and amelioration of osteoporosis in women : interaction of mechanical, hormonal and dietary factors. Sports

Cumming, D. C. (1996). Exercise-associated amenorrhea, low bone density, and estrogen replacement therapy. Archives of Internal Medicine, 156(19), 21932195.

De Crée, C. (1998). Sex steroid metabolism and menstrual irregularities in the exercising female. A review. Sports Medicine (Auckland, N.Z.), 25(6), 369-406.

De Souza, M. J., West, S. L., Jamal, S. A., Hawker, G. A., Gundberg, C. M., \& Williams, N. I. (2008). The presence of both an energy deficiency and estrogen deficiency 
497

498

499

500

501

502

503

504

505

506

507

508

509

510

511

512

513

514

515

516

517

518

519

exacerbate alterations of bone metabolism in exercising women. Bone, 43(1), 140-148. http://doi.org/10.1016/j.bone.2008.03.013

Di Masso, R. J., Font, M. T., Capozza, R. F., Detarsio, G., Sosa, F., \& Ferretti, J. L. (1997). Long-bone biomechanics in mice selected for body conformation. Bone, 20(6), 539-545.

Drinkwater, B. L., Nilson, K., Chesnut, C. H., Bremner, W. J., Shainholtz, S., \& Southworth, M. B. (1984). Bone mineral content of amenorrheic and eumenorrheic athletes. The New England Journal of Medicine, 311(5), 277-281. http://doi.org/10.1056/NEJM198408023110501

Golden, N. H. (2000). Osteoporosis prevention: a pediatric challenge. Archives of Pediatrics \& Adolescent Medicine, 154(6), 542-543.

Jepsen, K. J. (2011). Functional interactions among morphologic and tissue quality traits define bone quality. Clinical Orthopaedics and Related Research, 469(8), 2150-2159. http://doi.org/10.1007/s11999-010-1706-9

Jepsen, K. J., Evans, R., Negus, C. H., Gagnier, J. J., Centi, A., Erlich, T., ... Moran, D. S. (2013). Variation in tibial functionality and fracture susceptibility among healthy, young adults arises from the acquisition of biologically distinct sets of traits. Journal of Bone and Mineral Research: The Official Journal of the American Society for Bone and Mineral Research, 28(6), 1290-1300. http://doi.org/10.1002/jbmr.1879

Khosla, S. (2008). Estrogen and bone: Insights from estrogen-resistant, aromatasedeficient, and normal men. Bone, 43(3), 414-417. http://doi.org/10.1016/j.bone.2008.05.005 
520 Lane, N. E., Thompson, J. M., Haupt, D., Kimmel, D. B., Modin, G., \& Kinney, J. H.

521 (1998). Acute changes in trabecular bone connectivity and osteoclast activity in

Myerson, M., Gutin, B., Warren, M. P., Wang, J., Lichtman, S., \& Pierson, R. N. (1992). (Bethesda, Md.: 1985), 84(1), 37-46.

Loucks, A. B., Verdun, M., \& Heath, E. M. (1998). Low energy availability, not stress of exercise, alters LH pulsatility in exercising women. Journal of Applied Physiology Total body bone density in amenorrheic runners. Obstetrics and Gynecology, 79(6), 973-978.

Nattiv, A., Agostini, R., Drinkwater, B., \& Yeager, K. K. (1994). The female athlete triad. The inter-relatedness of disordered eating, amenorrhea, and osteoporosis. Clinics in Sports Medicine, 13(2), 405-418.

Nattiv, A., Loucks, A. B., Manore, M. M., Sanborn, C. F., Sundgot-Borgen, J., Warren, M. P., \& American College of Sports Medicine. (2007). American College of Sports Medicine position stand. The female athlete triad. Medicine and Science in Sports and Exercise, 39(10), 1867-1882. http://doi.org/10.1249/mss.0b013e318149f111

Ojeda, S. R., Wheaton, J. E., Jameson, H. E., \& McCann, S. M. (1976). The onset of puberty in the female rat: changes in plasma prolactin, gonadotropins, luteinizing hormone-releasing hormone (LHRH), and hypothalamic LHRH content. Endocrinology, 98(3), 630-638. http://doi.org/10.1210/endo-98-3-630 
543 Parfitt, A. M., Drezner, M. K., Glorieux, F. H., Kanis, J. A., Malluche, H., Meunier, P. J.,

$544 \quad$... Recker, R. R. (1987). Bone histomorphometry: standardization of nomenclature, symbols, and units. Report of the ASBMR Histomorphometry Nomenclature Committee. Journal of Bone and Mineral Research: The Official Journal of the American Society for Bone and Mineral Research, 2(6), 595-610.

Pettersson, U., Stålnacke, B., Ahlénius, G., Henriksson-Larsén, K., \& Lorentzon, R. (1999). Low bone mass density at multiple skeletal sites, including the appendicular skeleton in amenorrheic runners. Calcified Tissue International, 64(2), 117-125.

Pitukcheewanont, P., Austin, J., Chen, P., \& Punyasavatsut, N. (2013). Bone health in children and adolescents: risk factors for low bone density. Pediatric Endocrinology Reviews: PER, 10(3), 318-335.

Rakover, Y., Lu, P., Briody, J. N., Tao, C., Weiner, E., Ederveen, A. G., .. Ben-Shlomo, I. (2000). Effects of delaying puberty on bone mineralization in female rats. Human Reproduction (Oxford, England), 15(7), 1457-1461.

Rawlinson, S. C. F., Murray, D. H., Mosley, J. R., Wright, C. D. P., Bredl, J. C., Saxon, L. K., ... Pitsillides, A. A. (2009). Genetic selection for fast growth generates bone architecture characterised by enhanced periosteal expansion and limited consolidation of the cortices but a diminution in the early responses to mechanical loading. Bone, 45(2), 357-366. http://doi.org/10.1016/j.bone.2009.04.243 
565 Roth, C., Leonhardt, S., Seidel, C., Luft, H., Wuttke, W., \& Jarry, H. (2000).

566

567

568

569

570

571

572

573

574

575

576

577

578

579

580

581

582

583

584 585

586
Comparative analysis of different puberty inhibiting mechanisms of two GnRH agonists and the $\mathrm{GnRH}$ antagonist cetrorelix using a female rat model. Pediatric Research, 48(4), 468-474. http://doi.org/10.1203/00006450-200010000-00009

Roux, C., Kolta, S., Chappard, C., Morieux, C., Dougados, M., \& De Vernejoul, M. C. (1996). Bone effects of dydrogesterone in ovariectomized rats: a biologic, histomorphometric, and densitometric study. Bone, 19(5), 463-468.

Saine, M. E., Barbe, M. F., Agah, M. R., \& Yingling, V. R. (2011). Hypothalamic Suppression during Adolescence Varies by Bone Envelope: Medicine \& Science in Sports \& Exercise, 43(4), 608-616. http://doi.org/10.1249/MSS.0b013e3181f56a2c

Schally, A. V. (1970). Hypothalamic regulation of FSH and LH secretion. Research in Reproduction, 2(4), 2-3.

Schlecht, S. H., \& Jepsen, K. J. (2013). Functional integration of skeletal traits: an intraskeletal assessment of bone size, mineralization, and volume covariance. Bone, 56(1), 127-138. http://doi.org/10.1016/j.bone.2013.05.012

Sims, N. A., Morris, H. A., Moore, R. J., \& Durbridge, T. C. (1996). Increased bone resorption precedes increased bone formation in the ovariectomized rat. Calcified Tissue International, 59(2), 121-127.

Toft, M. F., Petersen, M. H., Dragsted, N., \& Hansen, A. K. (2006). The impact of different blood sampling methods on laboratory rats under different types of anaesthesia. Laboratory Animals, 40(3), 261-274. 
587 Warren, M. P., Brooks-Gunn, J., Fox, R. P., Holderness, C. C., Hyle, E. P., \& Hamilton, 588 W. G. (2002). Osteopenia in exercise-associated amenorrhea using ballet dancers as a model: a longitudinal study. The Journal of Clinical Endocrinology \& Metabolism, 87(7), 3162-3168.

Warren, M. P., Brooks-Gunn, J., Fox, R. P., Holderness, C. C., Hyle, E. P., Hamilton, W.

592

593

594

595

596

597

598

599

600

601

602

603

604

605

606

607

608

G., \& Hamilton, L. (2003). Persistent osteopenia in ballet dancers with amenorrhea and delayed menarche despite hormone therapy: a longitudinal study. Fertility and Sterility, 80(2), 398-404.

Warren, M. P., Brooks-Gunn, J., Fox, R. P., Lancelot, C., Newman, D., \& Hamilton, W. G. (1991). Lack of bone accretion and amenorrhea: evidence for a relative osteopenia in weight-bearing bones. The Journal of Clinical Endocrinology and Metabolism, 72(4), 847-853. http://doi.org/10.1210/jcem-72-4-847

Warren, M. P., \& Stiehl, A. L. (1999). Exercise and female adolescents: effects on the reproductive and skeletal systems. Journal of the American Medical Women's Association (1972), 54(3), 115-120, 138.

Wiggins, D. L., \& Wiggins, M. E. (1997). The female athlete. Clinics in Sports Medicine, 16(4), 593-612.

Wronski, T. J., Dann, L. M., \& Horner, S. L. (1989). Time course of vertebral osteopenia in ovariectomized rats. Bone, 10(4), 295-301.

Wronski, T. J., Dann, L. M., Scott, K. S., \& Cintrón, M. (1989). Long-term effects of ovariectomy and aging on the rat skeleton. Calcified Tissue International, 45(6), $360-366$. 
609 Yingling, V. R., \& Khaneja, A. (2006). Short-term delay of puberty causes a transient 610 reduction in bone strength in growing female rats. Bone, 38(1), 67-73.

$611 \quad$ http://doi.org/10.1016/j.bone.2005.07.001

612 Yingling, V. R., \& Taylor, G. (2008). Delayed pubertal development by hypothalamic 613 suppression causes an increase in periosteal modeling but a reduction in bone 614 strength in growing female rats. Bone, 42(6), 1137-1143.

$615 \quad$ http://doi.org/10.1016/j.bone.2008.02.005

616 Yingling, V. R., Xiang, Y., Raphan, T., Schaffler, M. B., Koser, K., \& Malique, R. (2007).

617 The effect of a short-term delay of puberty on trabecular bone mass and

618 structure in female rats: a texture-based and histomorphometric analysis. Bone, $61940(2), 419-424$. http://doi.org/10.1016/j.bone.2006.07.019

620 


\section{Figure 1 (on next page)}

Timeline of $\mathrm{GnRH}$-antagonist injection protocol, recover and ovariectomy surgery. 
Control

\begin{tabular}{|l|l|ll|l|l|l|}
\multicolumn{1}{c}{ C } & C-R & \multicolumn{2}{c}{ C-OVX } \\
\hline Control & $x$ & Recovery & $x$ & OVX & $x$ \\
$n=15$ & & $n=15$ & \\
$n$
\end{tabular}

GnRH-a

GnRH-a

G-R

G-OVX

\begin{tabular}{l|l|l} 
GnRH-a Injections & $x$ & Recovery
\end{tabular}



ovx

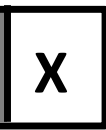

Age

$n=15$

$n=15$

$n=15$

(days)

25

50

185

225

$X=$ group euthanize 


\section{Figure 2 (on next page)}

Comparison of the timing of Vaginal Opening (VO) of all groups (C, GnRH-a, C-R, G-R, COVX, G-OVX).

The cumulative percent of animals with VO is displayed per age (days). The GnRH-a, G-R, GOVX groups have a lower percentage of animals with VO at later ages. The C, C-R and C-OVX groups reached $100 \%$ VO by day 39 . 


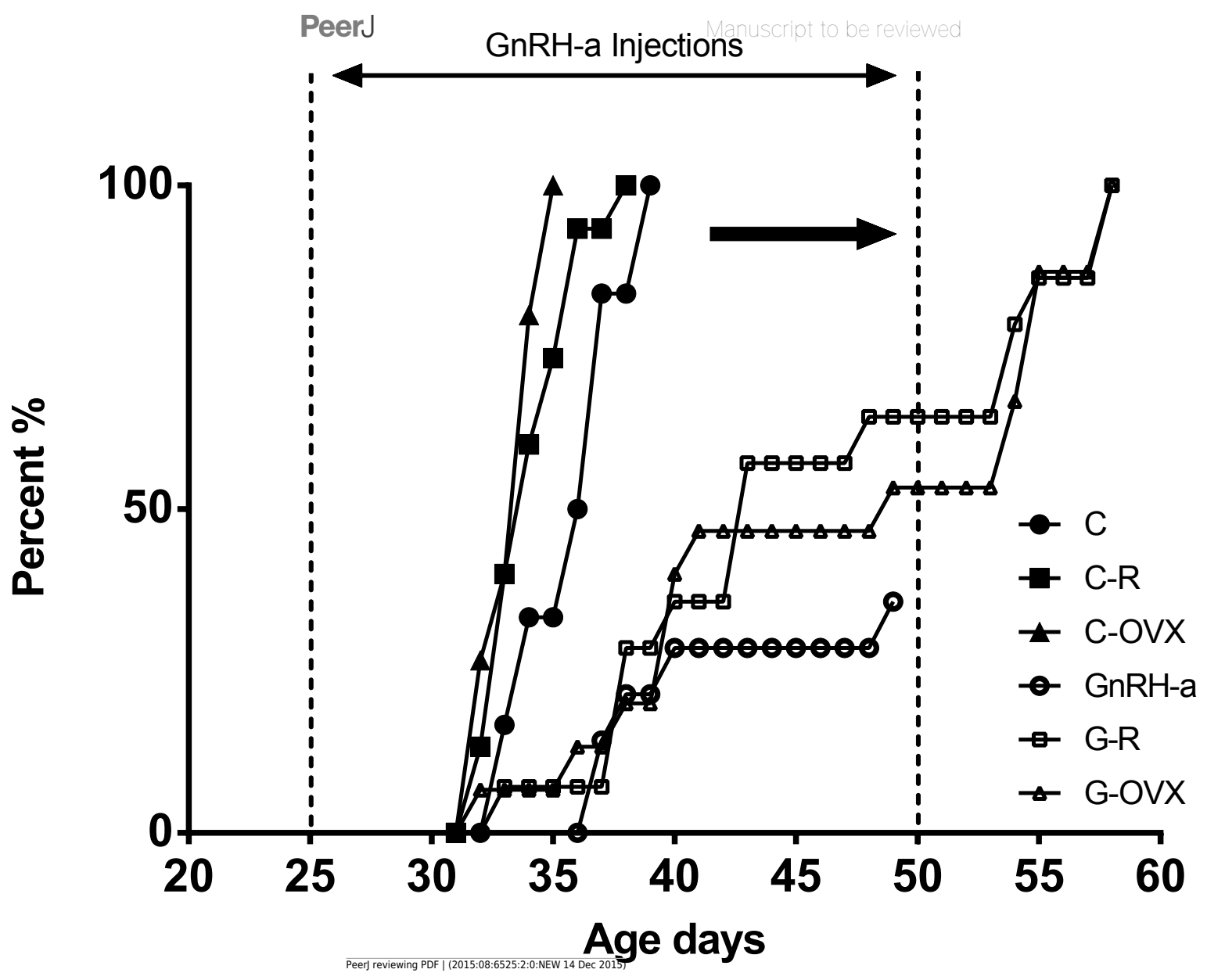




\section{Figure 3 (on next page)}

Uterine Weights, Growth Rates and Body Weights for the three time points, Injection Protocol, Recovery, post-OVX.
A) Uterine Weights (g) for C (black bars) and GnRH-a (white bars) groups, B) Growth Rate (g/week) for Early Puberty was calculated [(weight day 37-weight day 23)/2], Late Puberty was calculated [(weight day 50-weight day 37)/2]. C) Body Weight (g) at sacrifice for C (black bars) and GnRH-a (white bars) groups. ${ }^{*}$ Indicates significant difference from control values at same time point or from early puberty. " Indicates significant difference from Injection Protocol or from Control. ${ }^{\mathrm{v}}$ indicates significant difference from Recovery. 
PeÂR.

B.
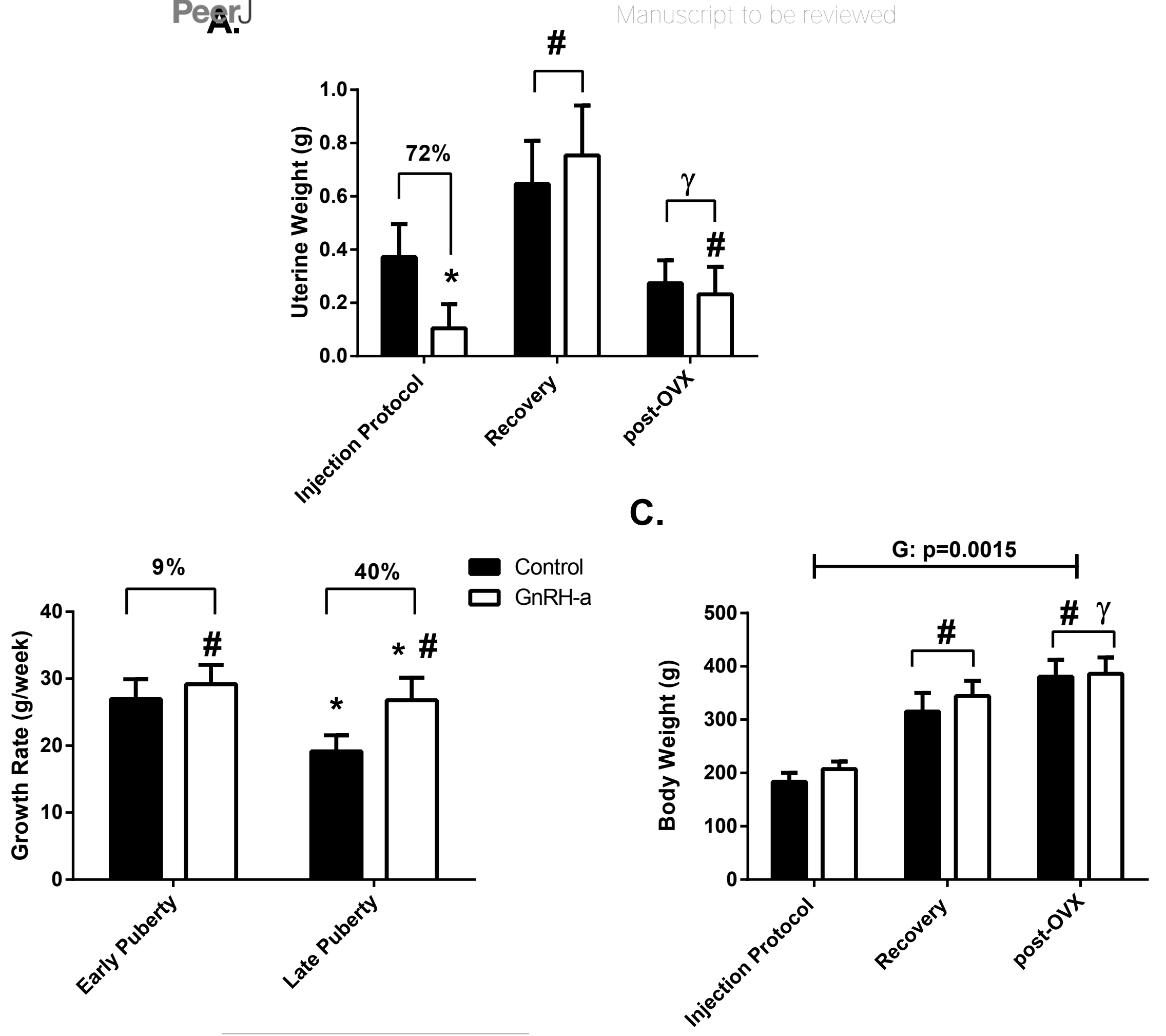


\section{Figure 4 (on next page)}

Serum markers of bone resorption (CTX) and formation (IGF-1).

A) serum CTX ( $\mathrm{ng} / \mathrm{mL}$ ) levels B) serum IGF-1 $\left(\mathrm{ng} / \mathrm{mL}\right.$ ) levels $(\mathrm{n}=8-10){ }^{*}$ Indicates significant difference from control values at same time point. * Indicates significant difference from Injection Protocol. ${ }^{\mathrm{r}}$ indicates significant difference from Recovery. 
A.

\section{Manuscript to be reviewed}

B.

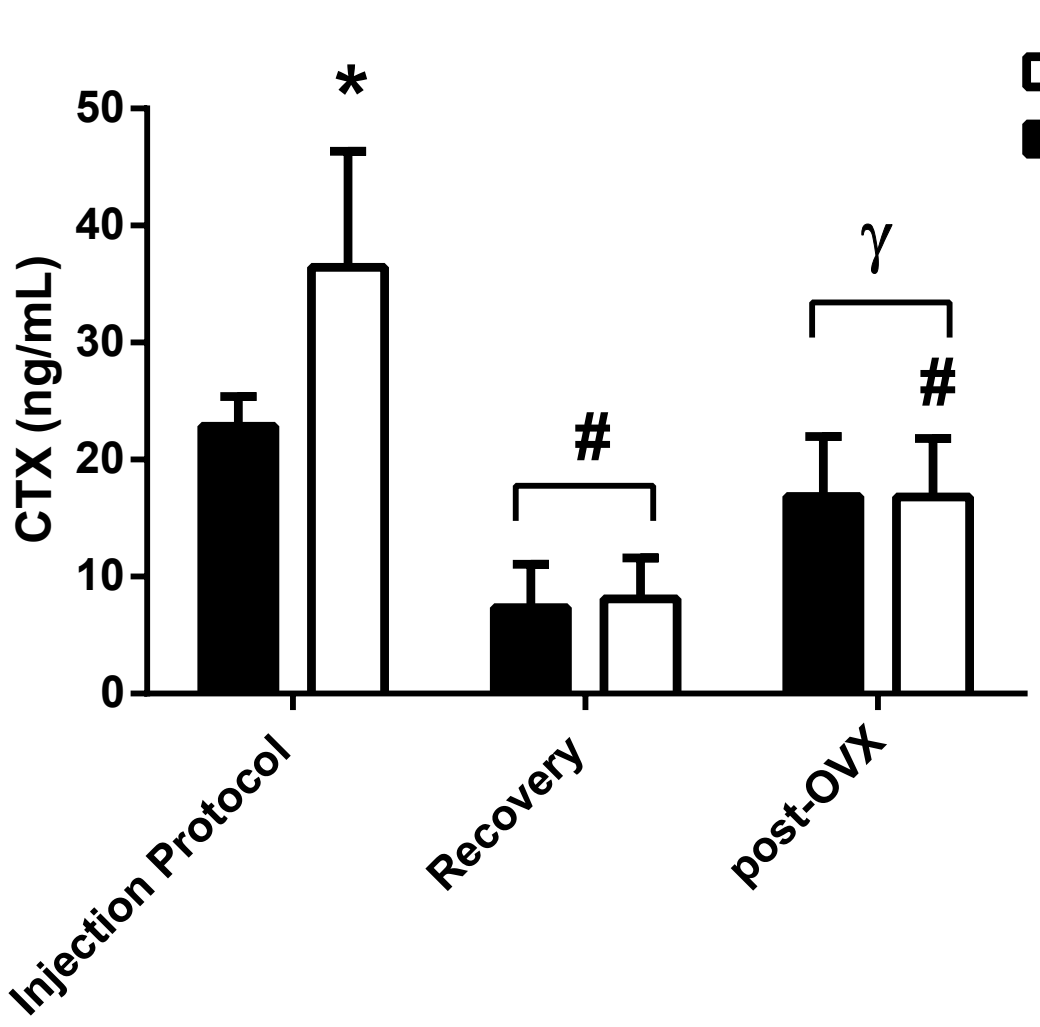

$\square \mathrm{GnRH}-\mathrm{a}$ Control

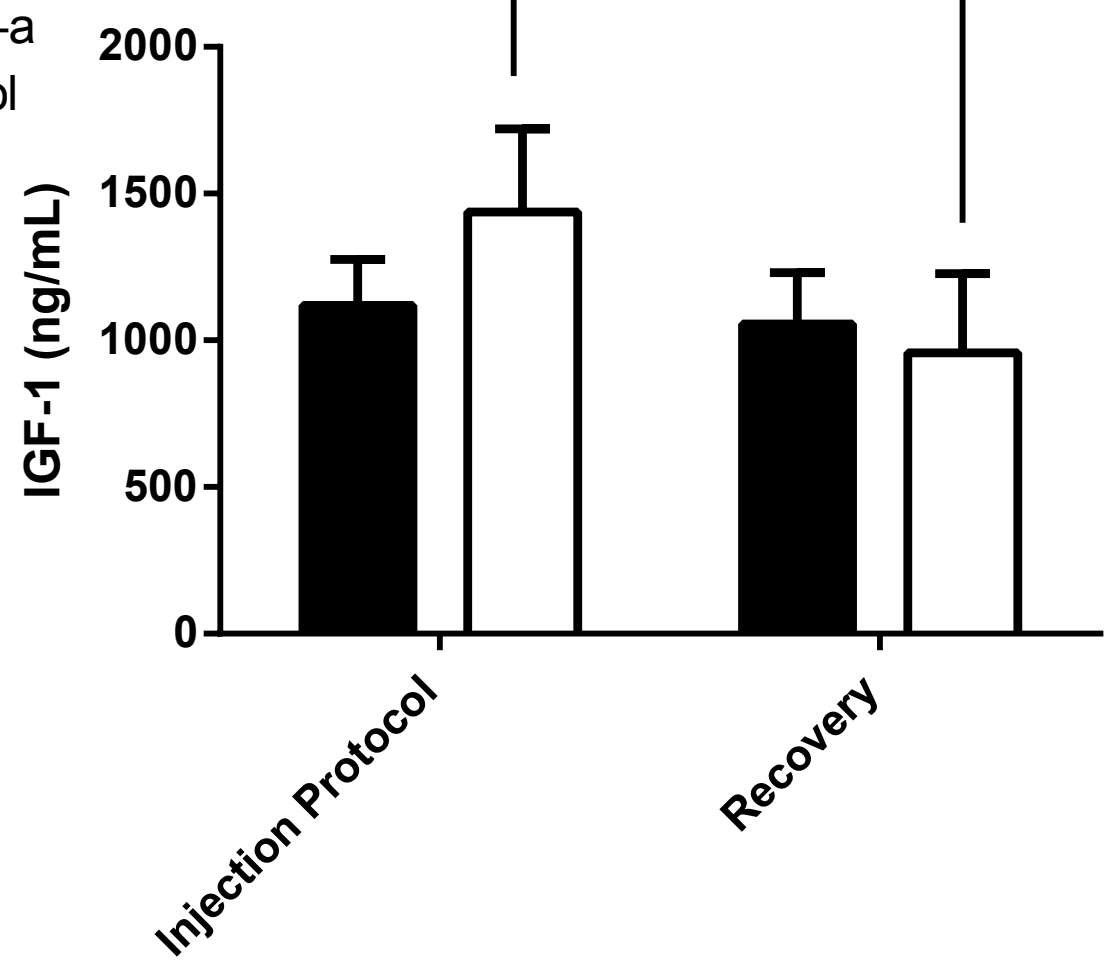




\section{Table $\mathbf{1}$ (on next page)}

Bone strength and structural parameters and body composition.

Whole bone mechanical parameters measured from three-point bending of the femoral and tibial diaphysis, geometry and structural measurements from micro CT analysis, bone and body composition measures. Mean (SD). ${ }^{*}$ Indicates significant difference from control values at same time point. " Indicates significant difference from Injection Protocol. ${ }^{v}$ indicates significant difference from Recovery. 


\begin{tabular}{|c|c|c|c|c|c|}
\hline & & $\begin{array}{l}\text { Injection } \\
\text { Protocol }\end{array}$ & Recovery & post-OVX & $\begin{array}{c}\text { 2-way ANOVA } \\
\text { p-values }\end{array}$ \\
\hline \multicolumn{6}{|l|}{ Mechanical Properties } \\
\hline \multirow{2}{*}{$\begin{array}{l}\text { Femur-Peak } \\
\text { Moment (N mm) }\end{array}$} & Control & $203 \pm 31$ & $557 \pm 140^{\# *}$ & $797 \pm 95^{\# \gamma}$ & \multirow[t]{2}{*}{ GxT: $p=0.0214$} \\
\hline & GnRH-a & $231 \pm 19$ & $713 \pm 130^{\#}$ & $823 \pm 90^{\# \gamma}$ & \\
\hline \multirow{2}{*}{$\begin{array}{l}\text { Femur-Stiffness } \\
\left(\mathrm{Nmm}^{2}\right)\end{array}$} & Control & $9880 \pm 2010$ & $42920 \pm 10880^{\#}$ & $73360 \pm 9490^{\# \gamma}$ & \multirow{2}{*}{$\begin{array}{l}G: p=0.0388 \\
T: p<0.0001\end{array}$} \\
\hline & GnRH-a & $9920 \pm 1240$ & $51400 \pm 13050^{\#}$ & $77010 \pm 8680^{\# \gamma}$ & \\
\hline \multirow{2}{*}{$\begin{array}{l}\text { Tibia- Peak } \\
\text { Moment (N mm) }\end{array}$} & Control & $189 \pm 17$ & $528 \pm 83^{\#}$ & $575 \pm 96^{\# \gamma}$ & \multirow[t]{2}{*}{$\mathrm{T}: \mathrm{p}<0.0001$} \\
\hline & GnRH-a & $197 \pm 18$ & $563 \pm 109^{\#}$ & $621 \pm 92^{\# \gamma}$ & \\
\hline \multirow{2}{*}{$\begin{array}{l}\text { Tibia-Stiffness } \\
\left(\mathrm{Nmm}^{2}\right)\end{array}$} & Control & $11003 \pm 1497$ & $50580 \pm 11488^{\#}$ & $57810 \pm 13240^{\# \gamma}$ & \multirow[t]{2}{*}{$\mathrm{T}: \mathrm{p}<\mathbf{0 . 0 0 0 1}$} \\
\hline & GnRH-a & $11650 \pm 1140$ & $56420 \pm 12980^{\#}$ & $62070 \pm 13040^{\# \gamma}$ & \\
\hline \multicolumn{6}{|l|}{ Geometry } \\
\hline \multirow[t]{2}{*}{$\mathrm{J}\left(\mathrm{mm}^{4}\right)$} & Control & $8.7 \pm 1.3$ & $13.1 \pm 1.8^{\#}$ & $15.8 \pm 2.3^{\# \gamma}$ & \multirow{2}{*}{$\begin{array}{l}G: p=0.0284 \\
T: p<0.0001\end{array}$} \\
\hline & GnRH-a & $9.3 \pm 1.0$ & $15.6 \pm 2.7^{\#}$ & $16.0 \pm 2.0^{\# \gamma}$ & \\
\hline \multicolumn{6}{|l|}{ Mass and Structure } \\
\hline \multirow[t]{2}{*}{ Ct.Ar $\left(\mathrm{mm}^{2}\right)$} & Control & $3.5 \pm 0.3$ & $6.2 \pm 0.4^{\#}$ & $6.8 \pm 0.4^{\# \gamma}$ & \multirow{2}{*}{$\begin{array}{l}\text { T: } \mathbf{p}<\mathbf{0 . 0 0 0 1} \\
\text { G: } p=0.0619\end{array}$} \\
\hline & GnRH-a & $3.8 \pm 0.2$ & $6.4 \pm 0.7^{\#}$ & $6.8 \pm 0.5^{\# \gamma}$ & \\
\hline \multirow[t]{2}{*}{ T.Ar $\left(\mathrm{mm}^{2}\right)$} & Control & $9.3 \pm 0.9$ & $9.4 \pm 0.8$ & $10.4 \pm 1.0^{\#}$ & \multirow[t]{2}{*}{$\mathrm{T}: \mathrm{p}<0.0001$} \\
\hline & GnRH-a & $9.3 \pm 0.8$ & $10.4 \pm 0.9$ & $10.4 \pm 0.7^{\#}$ & \\
\hline \multirow[t]{2}{*}{ Ma.Ar $\left(\mathrm{mm}^{2}\right)$} & Control & $5.8 \pm 0.9$ & $3.2 \pm 0.5^{\#}$ & $3.7 \pm 1.1^{\#}$ & \multirow[t]{2}{*}{$\mathrm{T}: \mathrm{p}<\mathbf{0 . 0 0 0 1}$} \\
\hline & GnRH-a & $5.5 \pm 0.8$ & $4.0 \pm 0.5^{\#}$ & $3.6 \pm 0.5^{\#}$ & \\
\hline \multirow[t]{2}{*}{ Ct.Th (mm) } & Control & $.22 \pm .03$ & $.52 \pm .12^{\#}$ & $.51 \pm .12^{\#}$ & \multirow[t]{2}{*}{$\mathrm{T}: \mathrm{p}<0.0001$} \\
\hline & GnRH-a & $.22 \pm .03$ & $.53 \pm .11^{\#}$ & $.60 \pm .05^{\#}$ & \\
\hline \multicolumn{6}{|l|}{ Bone Mineral Content } \\
\hline \multirow[t]{2}{*}{ Ash Fraction \% } & Control & $58 \pm 4$ & $67 \pm 1$ & $65 \pm 2$ & \multirow[t]{2}{*}{$\mathrm{T}: \mathrm{p}<0.0001$} \\
\hline & GnRH-a & $60 \pm 2$ & $66 \pm 2$ & $65 \pm 5$ & \\
\hline \multicolumn{6}{|l|}{ Body Composition } \\
\hline \multirow{2}{*}{$\begin{array}{l}\text { Triceps Surae Muscle } \\
\text { Mass/ BW (\%) }\end{array}$} & Control & $0.65 \pm 0.04$ & $0.67 \pm 0.06$ & $0.63 \pm 0.05^{\gamma}$ & \multirow[t]{2}{*}{ GxT: $p=0.0455$} \\
\hline & GnRH-a & $0.62 \pm 0.03$ & $0.68 \pm 0.04^{\#}$ & $0.66 \pm 0.05^{\#}$ & \\
\hline \multirow[t]{2}{*}{ Total Fat Pads (g) } & Control & $1.2 \pm 0.6$ & $10.0 \pm 4.3^{\#}$ & $12.6 \pm 3.6^{\#}$ & \multirow{2}{*}{$\begin{array}{l}\text { T: } \mathbf{p}<\mathbf{0 . 0 0 0 1} \\
\text { G: } p=0.0640\end{array}$} \\
\hline & GnRH-a & $2.0 \pm 0.7$ & $12.4 \pm 2.7^{\#}$ & $13.8 \pm 4.3^{\#}$ & \\
\hline
\end{tabular}

* Indicates significant difference from control values at same time point. 
3

\# Indicates significant difference from Injection Protocol.

$\gamma$ indicates significant difference from Recovery. 
Figure $\mathbf{5}$ (on next page)

Structural measurements from micro CT analysis.

A) Percent trabecular bone volume (BV/TV) (\%), B) Trabecular Number (Tb.N) (1/mm), C) Trabecular Thickness (Tb.Th) (mm), D) Trabecular Separation (Tb.Sp) (mm) ( $n=9-15)$ * Indicates significant difference from control values. " Indicates significant difference from Injection Protocol. ${ }^{v}$ indicates significant difference from Recovery. 
A.

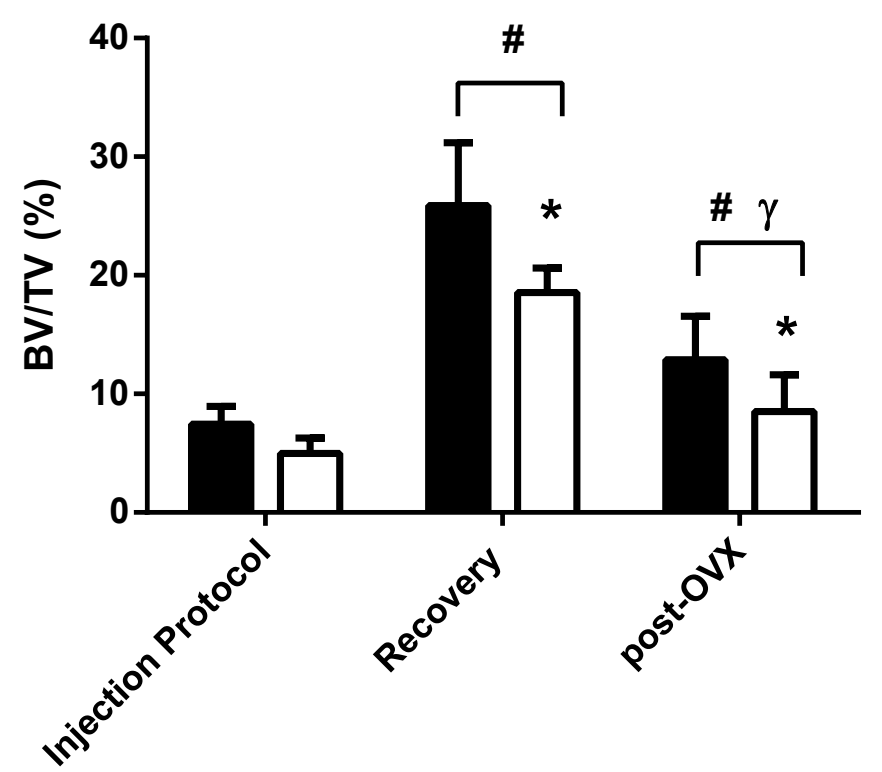

C.

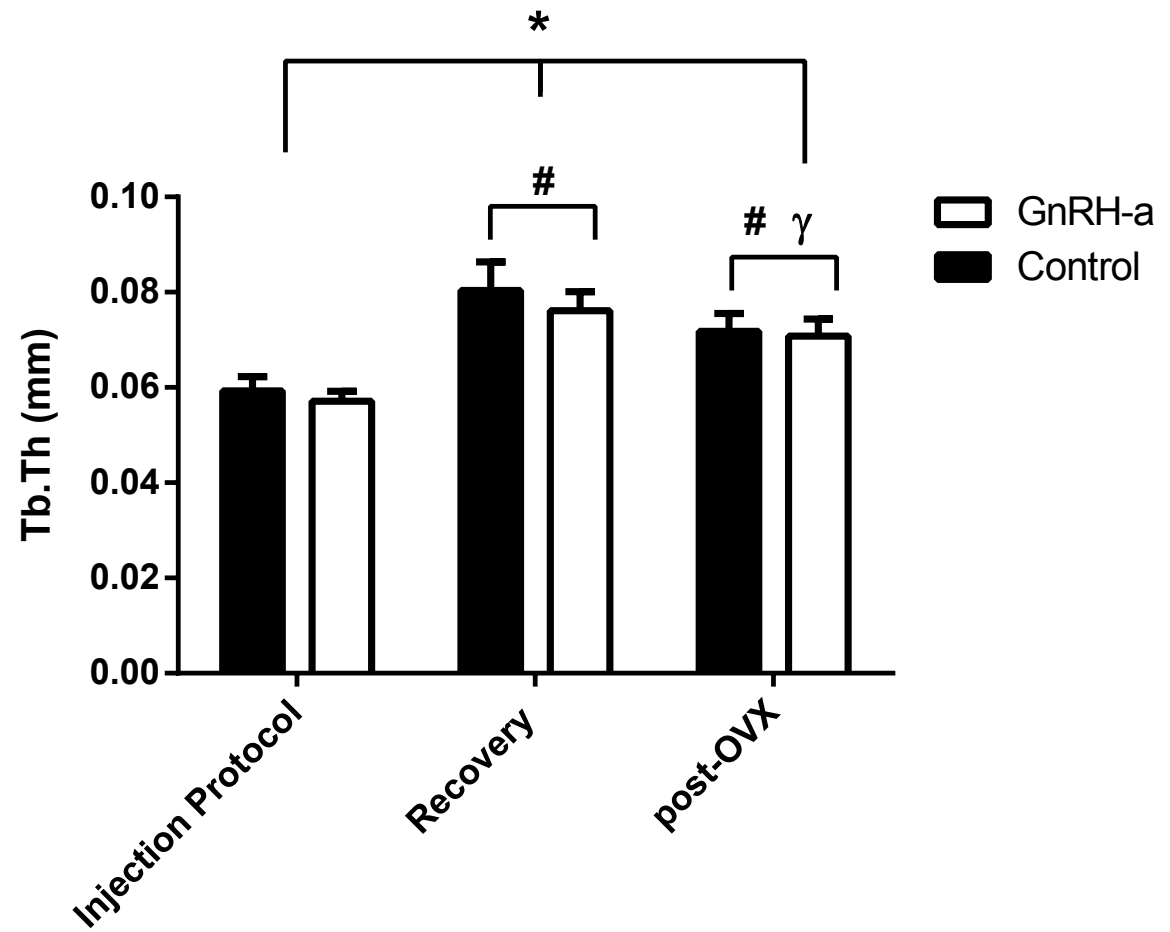

Manuscript to be reviewed *

B.
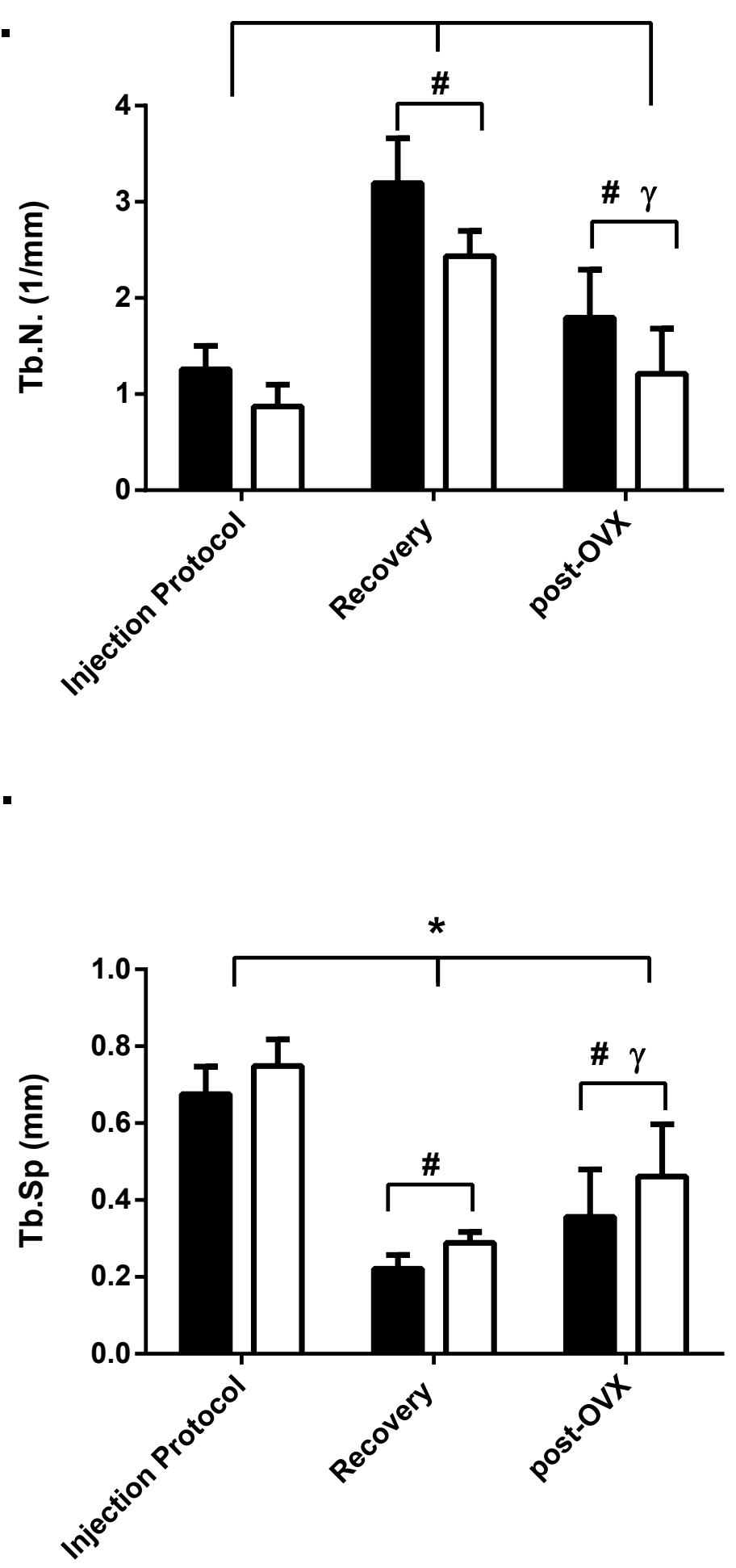
Figure $\mathbf{6}$ (on next page)

Linear regression of of body weight and bone structural variables for the GnRH-a and control groups.

A) There was a significant relationship between body weight and polar moment of inertia but no difference between groups ( $\mathrm{C}: \mathrm{R}^{2}=.9188 ; \mathrm{GnRH}-\mathrm{a}$ : $\mathrm{R}^{2}=.7846$ ). B) There was a significant relationship between body weight and cortical area but no difference between groups (C: $\mathrm{R}^{2}=.8909 ; \mathrm{GnRH}-\mathrm{a}: \mathrm{R}^{2}=.9079$ ). C) There was a significant relationship between polar moment of inertia and cortical area but no difference between groups ( $C$ : $R^{2}=.7893$; $G n R H-a$ : $\left.\mathrm{R}^{2}=.9043\right)$. 

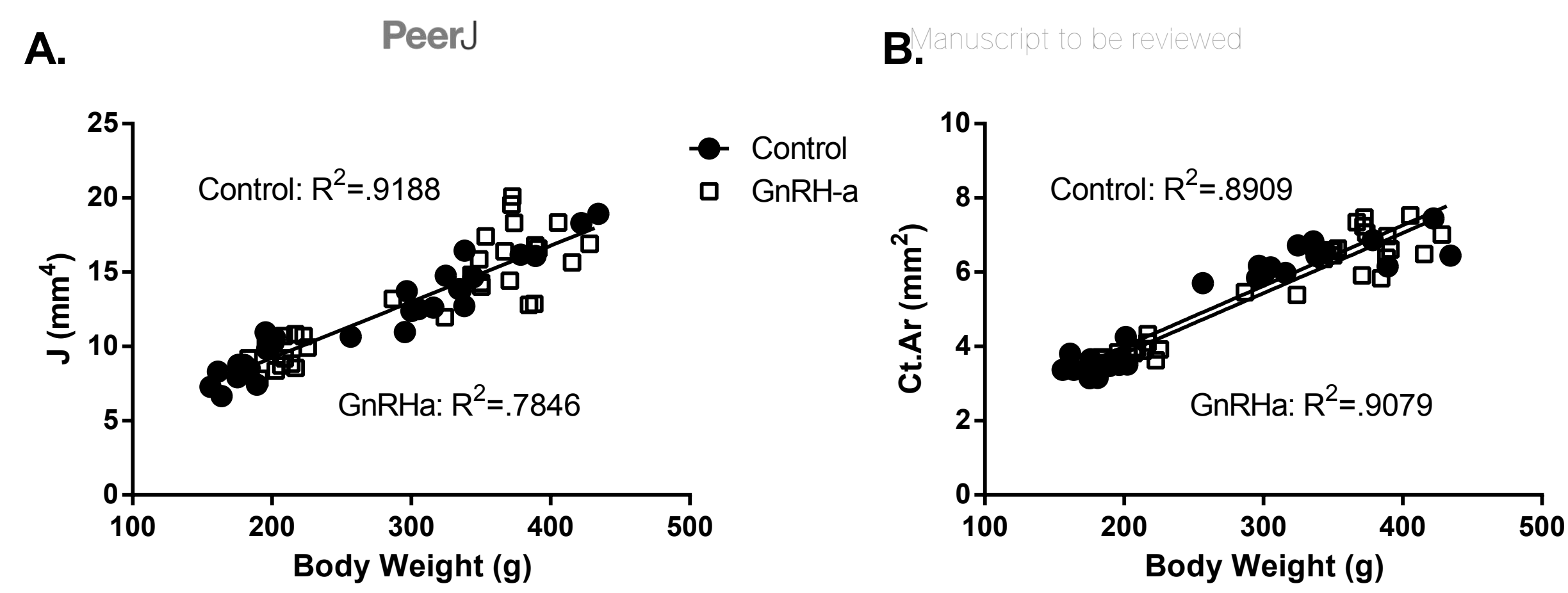

C.

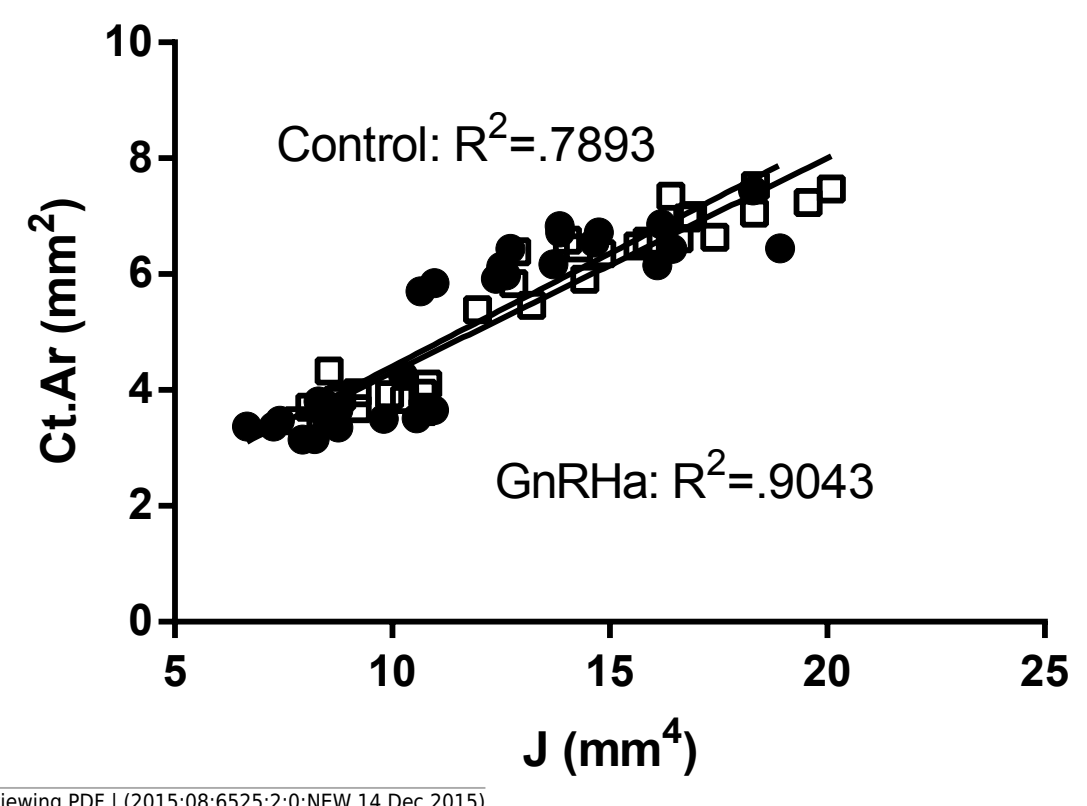

- Control

— GnRH-a 